Portland State University

PDXScholar

2014

\title{
Asymmetry of Matter/Antimatter and Possibility of CPT Violations
}

Kai Pak

Portland State University

Follow this and additional works at: https://pdxscholar.library.pdx.edu/honorstheses

Let us know how access to this document benefits you.

Recommended Citation

Pak, Kai, "Asymmetry of Matter/Antimatter and Possibility of CPT Violations" (2014). University Honors Theses. Paper 39.

https://doi.org/10.15760/honors.81

This Thesis is brought to you for free and open access. It has been accepted for inclusion in University Honors Theses by an authorized administrator of PDXScholar. Please contact us if we can make this document more accessible: pdxscholar@pdx.edu. 


\title{
Asymmetry of Matter/Antimatter and Possibility of CPT Violations
}

\author{
by \\ Kai Pak
}

An undergraduate honors thesis submitted in partial fulfillment of the

requirements for the degree of

Bachelor of Science

in

University Honors

and

Physics

Thesis Advisor

Jack C. Straton

Portland State University

2014 


\begin{abstract}
Symmetries are an important area of study in physics, fundamental to the understanding of all aspects of Nature including the beginning and formation of the Universe, and how it appears to us today. While current quantum theories and experiments observe that CPT (Charge Conjugation, Parity, and Time Reversal) symmetry is conserved, there are speculations that this may not be the case with matter and antimatter and could provide answers as to why such a large asymmetry (also known as baryogenesis) exists between the two with normal matter completely dominating the observable Universe. Key to studying antimatter is the ability to properly capture it in low enough energy states where accurate experiments can be conducted. To assist with experimentalists' efforts at CERN to confine antihydrogen, calculations have determined the probabilities for producing antihydrogen ions by the most efficient means that can be used to ultra-cool antihydrogen (mK-range), and allow detailed study.
\end{abstract}




\section{Dedication}

To dad. Without your inspiration, I would have given up on school long ago. You are missed. 


\section{Acknowledgements}

I would like to express my warmest thanks to my undergraduate advisor, Professor Jack C. Straton. He has been a wonderful mentor and helped me grow tremendously as a researcher and physicist. I continue to be astounded by his brilliance and his ability to inspire excitement and curiosity in exploring the Universe.

I would also like to extend thanks to Chris Keating, and Professor Mike Charlton for their invaluable insight and input on this thesis.

Finally, I would like to thank Greg Bergs, my director at work. He encouraged me to pursue my degree in physics at Portland State University while working on his team and provided me the support and flexibility I needed to successfully juggle a full-time career while following my educational dreams. 


\section{Contents}

1 The Discovery of Antimatter $\quad 7$

1.1 A Strange Man . . . . . . . . . . . . . . . . 7

1.2 The Klein-Gordon Equation . . . . . . . . . . . . . . 8

1.3 The Dirac Equation . . . . . . . . . . . . . 9

2 Symmetries 12

2.1 Practical Examples . . . . . . . . . . . . . . . . . 12

2.2 Noether's Theorem and Conservation Laws . . . . . . . . 13

2.3 Breaking Symmetries . . . . . . . . . . . . . . . . . . 14

2.4 Pion Decay and the Fall of Parity (P) Symmetry . . . . . . . 15

2.5 The Fall of CP (Charge Conjugation/Parity) Symmetry . . . 18

2.6 Time and CPT (Charge Conjugation/Parity/Time) Symmetry 19

3 Capturing Antimatter $\quad 21$

3.1 Where is all the Antimatter? . . . . . . . . . . . . . . 21

3.2 Technical Hurdles . . . . . . . . . . . . . . . . . . . . . . . 22

3.3 Current Work ...................... 24

4 Continuing Search for CPT Asymmetry 27

4.1 CPT Tests and the Consensus Thus Far . . . . . . . . . . 27

4.2 Future Studies . . . . . . . . . . . . . . . . . . . . . . . . . . . . . . . . 28

4.3 Conclusion ........................... 28

A The Dirac Equation: How Matrices Save the Day 30

B Noether Theorem: Linear One-Dimensional Case 32 
C Photodetachment and Radiative Attachment 34

C.1 Photodetachment . . . . . . . . . . . . . . . 34

C.2 Radiative Attachment . . . . . . . . . . . 35 


\section{List of Figures}

1.1 Carl D. Anderson electron/positron emission plate . . . . 10

2.1 Examples of symmetry . . . . . . . . . . . . . . . . 12

2.2 Clock/Anti-clock example . . . . . . . . . . . . . . . 15

2.3 Wu Experiment on Cobalt-60 Decay . . . . . . . . . . . . 16

2.4 Cobalt-60 Parity Violation Diagram . . . . . . . . . . 17

3.1 Pulsar (rapidly rotating neutron star) located the heart of the Crab Nebula . . . . . . . . . . . . . . . . 22

3.2 ALPHA Central Apparatus . . . . . . . . . . . . . 24

3.3 Cross section of Photodetachment of Electron from Hydrogen Forming $H^{-} \ldots \ldots \ldots \ldots . \ldots \ldots$

3.4 Cross Section of Radiative Attachment of Second Positron Forming $\bar{H}^{+} \ldots \ldots \ldots \ldots \ldots \ldots$ 


\section{Chapter 1}

\section{The Discovery of Antimatter}

\subsection{A Strange Man}

Antimatter strikes those learning about it for the first time as something mysterious, other-worldly, and altogether strange. The story of its discovery is perhaps, stranger still. It begins not with an accidental eureka moment in some advanced laboratory; but simply, with a theoretician wielding pen and paper. P.A.M. Dirac was rather eccentric even by physicist standards ${ }^{1}$. It is quite fitting that he would be the man whose theoretical work would require the existence of the bizarre antiparticle yang version to every particle's yin-even before it was ever observed in Nature.

The equation that bears Dirac's name stands as one of the great works of theoretical physics that finally merged Einstein's special relativity with Schrödinger's quantum wave equation into a single cohesive edifice. With mathematical brilliance and physical intuition, Dirac was able to fully provide a theoretical justification for electron spin as described by Pauli, successfully create a Lorentz invariant quantum equation, and as an added result, imply the existence of antiparticles. However, at the time, the last implication was not entirely clear and indeed puzzled Dirac for some time.

\footnotetext{
${ }^{1}$ According to one account, when a classmate at St. Johns College off-handedly said, "it's a bit rainy, isn't it?", Dirac responded by standing up, walking over to the window, then replying, "it is not raining now." [14]
} 


\subsection{The Klein-Gordon Equation}

In the realm of non-relativistic quantum mechanics, the existing framework, based primarily on the Schrödinger wave equation, provided numerous experimentally confirmed results and had successfully modeled the hydrogen atom to high degrees of accuracy. However, applying these equations to particles approaching relativistic speeds $(\geq .10 \mathrm{c})$, leads to increasingly invalid results.

The first well-known attempt at a solution was the Klein-Gordon equation. Essentially, the idea was to start with Einstein's relativistic energy equation:

$$
E^{2}=p^{2} c^{2}+m^{2} c^{4}
$$

and drop in momentum and energy operators from the Schrödinger wave equation:

$$
\nabla^{2}-\frac{1}{c^{2}} \frac{\partial^{2}}{\partial t^{2}}=\frac{m^{2} c^{2}}{\hbar^{2}}
$$

The immediate issue with this equation is that it is not linear with time as the squaring of energy leads to second order partial differential equations which - in addition to being difficult to solve analytically-is inconsistent with Schrödinger's wave equation (which is linear in time). Moreover, it is wholly inconsistent with Born's statistical interpretation (demanding $|\psi(r)|^{2}$ gives the probability of finding a particle at point $r$ ), in that the Klein-Gordon equation gives rise to negative probability densities [19]. Another major flaw is that it doesn't allow for spin states (other than 0 ) which manifests in the failure of the equation to correctly produce the Bohr energy states of hydrogen [19].

Finally, due to the squaring of energy, the Klein-Gordon equation allowed for negative solutions:

$$
E= \pm \sqrt{p^{2} c^{2}+m^{2} c^{4}}
$$

At the time, this was viewed the fatal blow to the equation and deemed non-physical. As will later be seen, however, the implications were actually not a problem after all.

Although ultimately flawed, the Klein-Gordon equation was a significant step in the development towards producing a quantum wave equation consistent with special relativity. It would find later usefulness in 
some field theories describing particles of spin 0, but as an all-purpose relativistic quantum equation describing one particle, it was not a good candidate.

\subsection{The Dirac Equation}

Due to these failures, Dirac set out to find a solution. The primary issue with the Klein-Gordon equation was due to the squaring of the Hamiltonian. Thus, Dirac dispensed with that starting point altogether and hit upon the idea of introducing new variables and taking the square root of the Klein-Gordon equation, thereby maintaining a first order equation consistent with Schrödinger, as well as Born's statistical interpretation:

$$
\nabla^{2}-\frac{1}{c^{2}} \frac{\partial^{2}}{\partial t^{2}}=\left(A \partial_{x}+B \partial_{y}+C \partial_{z}+\frac{i}{c} D \partial_{t}\right)^{2},
$$

which, when combined with the right side of Eq. 1.2, leads to,

$$
A \partial_{x}+B \partial_{y}+C \partial_{z}+\frac{i}{c} D \partial_{t}-\frac{m c}{\hbar}=0
$$

Dirac's great insight was to realize that the included variables $(A, B, C, D)$ could not be scalars - they had to be $4 \times 4$ matrices. $^{2}$ With $A=i \beta \alpha_{1}, B=$ $i \beta \alpha_{2}, C=i \beta \alpha_{3}, D=\beta$, where $\alpha$ and $\beta$ are matrices, and $\gamma^{0}=\beta, \gamma^{k}=\gamma^{0} \alpha^{k}$; in atomic units, the equation takes the simplified form of,

$$
i \gamma^{u} \partial_{u} \psi=m \psi
$$

where we sum over the repeated index $u$.

The Dirac Equation seemed to fix most of the shortcomings of the Klein-Gordon equation, but still allowed for the puzzling negative solutions. Because of this, initial reaction to Dirac's equation was decidedly icy with Heisenberg saying "the saddest chapter of modern physics is and remains the Dirac theory" [26]. To counter these criticisms, Dirac proposed a somewhat bizarre hypothesis known as the "hole theory" where the negative states represent a sea of electrons that are always occupied. Due to the Pauli exclusion principle, he argued, electrons could never attain these states, and that any unoccupied eigenstate (which he dubbed a

\footnotetext{
${ }^{2}$ See appendix A for more a more detailed explanation of how matrices solved the problem.
} 


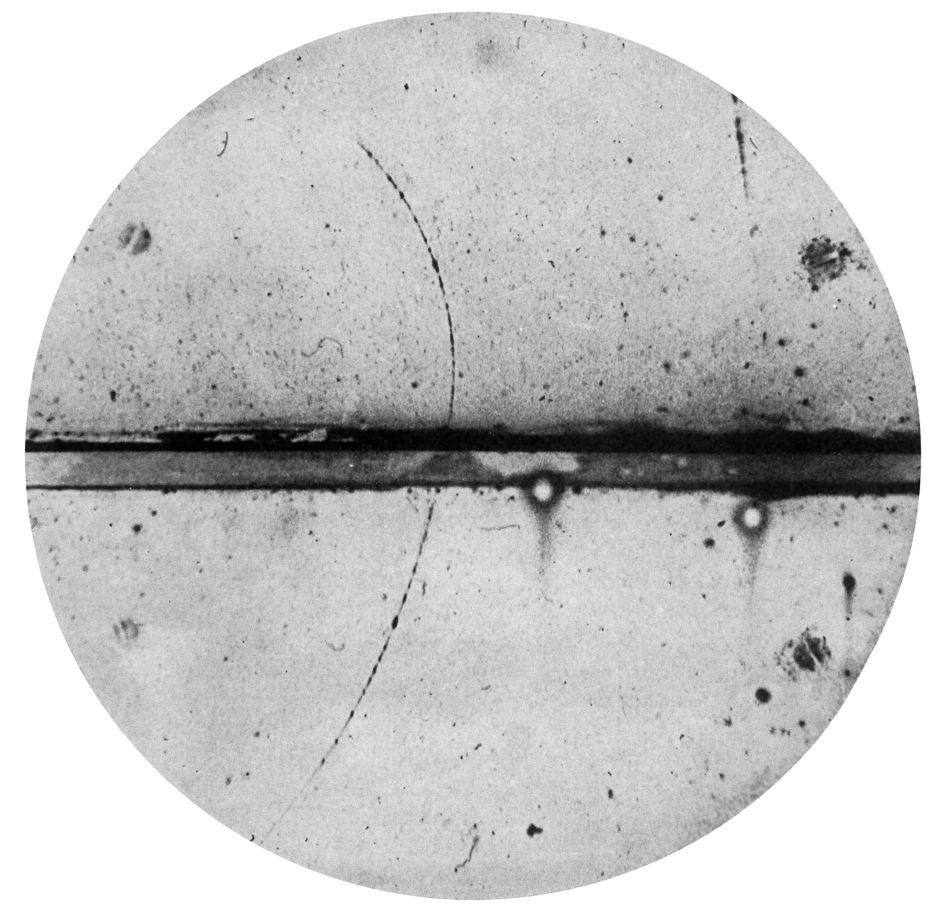

Figure 1.1: Cloud chamber detection of electron/positron emission by Carl D. Anderson at Caltech. The lines curving away from the horizontal line bisecting the image show the diverging paths of the electron/positron pairs.

"hole") must have positive charge and might possibly represent positively charged protons [22].

Some have suggested that he was merely joking with this interpretation [8]; but, whatever Dirac's actual original beliefs, Oppenheimer convincingly argued that the "hole" could not possibly be a proton due to the large known difference in mass between it and electrons, and concluded that it had to be a particle of similar mass but positive charge. Dirac eventually embraced this idea and included it in his paper: thus, the idea of the positron-the antiparticle to the electron-was born [22,27].

Dirac's validation would arrive with Carl D. Anderson at Caltech in 1932. An experimentalist examining cosmic rays in a cloud chamber, Anderson discovered paths of electrons with identical mass but opposite charge. Upon further investigation, it became clear that he was observing the positron as predicted by Dirac.

Dirac firmly believed that symmetries are fundamental to Nature, and 
that the now proven existence of antiparticles must, by necessity, suggest the existence of large amounts of antimatter elsewhere in the Universe. In his Nobel acceptance speech, he affirmed this belief:

If we accept the view of complete symmetry between positive and negative electric charge so far as concerns the fundamental laws of Nature, we must regard it rather as an accident that the Earth (and presumably the whole solar system), contains a preponderance of negative electrons and positive protons. It is quite possible that for some of the stars it is the other way about, these stars being built up mainly of positrons and negative protons. In fact, there may be half the stars of each kind. The two kinds of stars would both show exactly the same spectra, and there would be no way of distinguishing them by present astronomical methods [10].

An outcome of matter and antimatter interaction is annihilation and the release of electromagnetic radiation. Presumably, if large quantities of antimatter exist in the universe, we could detect them by the gamma radiation that would be pouring out at the borders where the antimatter galaxies ended and matter galaxies began. In the decades since its discovery however, we have been unable to find such large quantities of antimatter. Despite our efforts and no matter where and how far we look, we cannot seem to find antimatter planets, stars, solar systems, or galaxies. The current consensus among cosmologists is that such large amounts of antimatter simply do not exist in the Universe.

Considering the notion of symmetries is what led Dirac down the path to creating the mathematical formulation for antiparticles, and the fact that these equations were proven correct by experiment, it begs the question: where is all the antimatter? 


\section{Chapter 2}

\section{Symmetries}

\subsection{Practical Examples}

As humans are bilaterally symmetric, intrinsically, we know that this means we can be cut into left and right halves and that we are (mostly) invariant from either side. Another way to put this would be to say that either half of us held up to a mirror would reproduce the full image (with, perhaps, very oddly parted hair). Observe the images in figure 2.1 and note that levels of symmetry are increasing until we get to the circle: no matter what direction you bisect or rotate it, its mirror half will always produce the same image: it is infinitely symmetric.
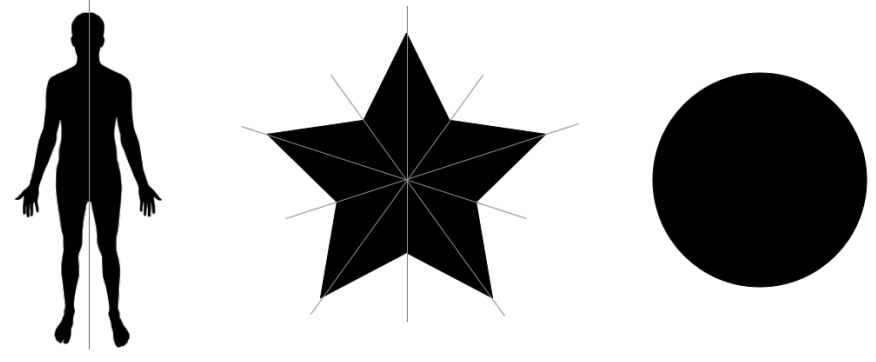

Figure 2.1: A few examples of symmetry. The man displays bilateral symmetry, but not rotational symmetry. The star displays several axes of symmetry and is also rotationally symmetric (A $72^{\circ}$ rotation in either direction will produce the same star). The circle has infinite axes of symmetries and is also infinitely rotationally symmetric.

Perhaps it's a little surprising that the Universe itself seems to also pre- 
fer symmetries. Symmetries in physics are more rigorously defined and subtle, but they have an analogous meaning in comparison to the shapes used in our simple example. Just as we derive symmetries by noting invariance among parts (e.g. the man is invariant left to right), physicists derive symmetries by finding invariance amongst initial physical conditions. The symmetries themselves lead to even deeper implications about how the Universe works.

\subsection{Noether's Theorem and Conservation Laws}

Symmetries have become a dominant cornerstone of modern quantum theories and are a reoccurring theme that permeates natural sciences and mathematics. However, while symmetry was seen as an important feature of nature, it was viewed as a mere consequence of dynamical laws. In 1918, Emmy Noether would prove a much deeper connection and that symmetries actually define conservation laws. In essence ${ }^{1}$, Noether's theorem proved that symmetry in translation of space means linear momentum is conserved; symmetry of rotation means angular momentum is conserved; and symmetry of translation in time means energy is conserved. Physicists had long ago noted that a variance in time or space does not change the fundamental way in which the laws of the Universe behave. For example, the laws governing the motion of a car driving down the road do not rely on where or when you are: they are identical in Timbuktu in the $21^{\text {st }}$ century as they will be on a moon colony in the $25^{\text {th }}$ century. What Noether did was explicitly prove mathematically that these symmetries are not a byproduct of conservation laws, they are the source.

To put it plainly, physics could not exist in its current form without the existence of these fundamental symmetries. Symmetries mean there is a framework of predictability in the Universe that is independent of initial conditions of space, rotation, and time. If this were not so, the Universe would be hopelessly complex and we would find great difficulty in generalizing laws from one place or moment to another: Newton's laws of motion would falter depending on if you were in Portland, Oregon or Paris, France; we'd have to construct different laws that separately describe the rotations of Mars and Earth around the Sun; and the laws discovered by

\footnotetext{
${ }^{1}$ See appendix B for a simplified derivation of conservation of linear momentum from symmetry of one-dimensional translation in space.
} 
earlier scientists would differ from the laws we find today if Nature were not invariant under time. Fortunately, not only is the Universe symmetric in these fundamental ways, but it also exhibits similarities between disparate phenomena. Symmetries that apply to planets orbiting a star also apply to the electrons and protons of the subatomic realm. To wit, if you can describe one electron, you describe them all.

The contributions to physics Noether's theorem has made are profound. ${ }^{2}$. A strong case can be made that her work is the foundation on which all modern physics is based. In fact, symmetries have proven to be even more important to quantum theories than in classical physics. It would not be an exaggeration to say that all of modern physics is based on a foundation of symmetries. In fact, it is the notion of symmetries that first clued Dirac and others in to the idea that every particle must have a complementary antiparticle and was the answer to the mysterious negative solutions that arose out of his equation.

\subsection{Breaking Symmetries}

Consider a mechanical clock in all its intricacies with gears, screws, bolts, springs, etc. Now, imagine that we build an identical clock, but in reverse: the screws tighten counter clockwise, the gears spin backwards, and the numbers on the face are reversed. Let us call this an anti-clock. What would be expected to happen? We should assume that the physical laws that govern the anti-clock remain the same and that it would behave identically to the ordinary clock, only in reverse.

The anti-clock would tick counterclockwise but should show the correct time. In physics parlance, we say that the anti-clock is symmetric and that it is invariant under a mirror translation (parity). What would be peculiar is if the anti-clock continued to rotate clockwise despite the reverse orientation of its mechanics (Fig. 2.2). This is known as a parity violation.

Up until 1956, physicists believed there were no such violations in Nature - that in fact, the Universe was built in a way that is so fundamentally symmetric, all phenomena, whether it be electrons, planets, or hypothetical clocks obeyed parity. Note that the parity obeying clock/anti-clock

\footnotetext{
${ }^{2}$ In Noether's obituary, Einstein wrote that she, "was the most significant creative mathematical genius thus far produced since the higher education of women began" [13] The fact that Noether did not (and continues to not) receive greater recognition is one of the great injustices in the history of science.
} 

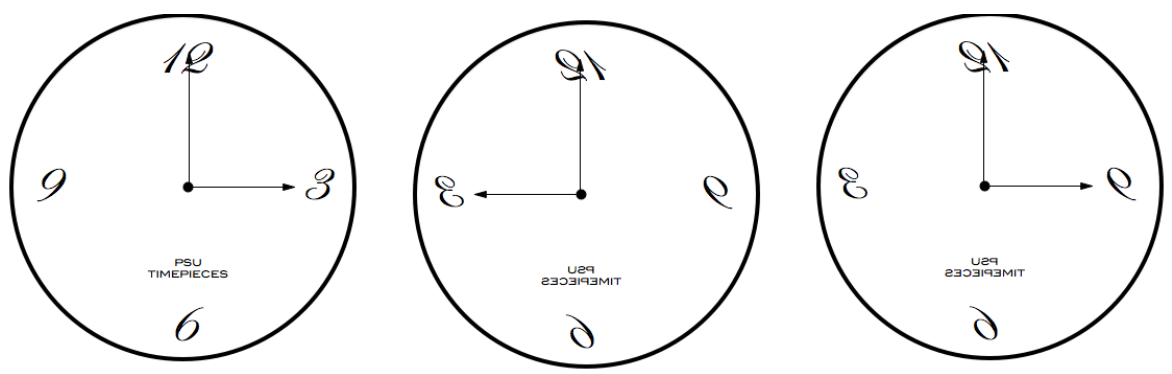

Figure 2.2: From left to right, a clock, symmetric anti-clock, and symmetry breaking anticlock. Note, that although all the parts in the symmetric anti-clock have been reversed, it is still showing the correct time. Conversely, the symmetry breaking anti-clock has all parts reversed but displays the wrong time-it appears to favor a direction of rotation.

shows no preference of handedness: the clock turns to the right, while the anti-clock turns left. It was taken as fact that this is how Nature designed itself as well-that it too was ambidextrous. For strong forces on the human up to galactic scale, this turns out to be true as far as we can see. Electromagnetism, gravitation, and strong nuclear forces: these all obey laws of symmetry and a single exception has never been found.

\subsection{Pion Decay and the Fall of Parity (P) Symmetry}

Doubts of the absolute laws of symmetry would only arise with the rapid development of modern quantum theories and the discovery of weak forces that are responsible for radioactive decay and other subatomic particle interactions. Among the first clues that parity might be broken in the weak interactions occurred with the study of the so-called " $\tau-\theta$ (tau-theta) puzzle." In this decay process, two K-mesons (or kaons: subatomic particles comprised of one quark and one antiquark), were observed to decay in different ways:

$$
\begin{aligned}
& \theta^{+} \rightarrow \pi^{+}+\pi^{0} \\
& \tau^{+} \rightarrow\left\{\begin{array}{l}
\pi^{+}+\pi^{0}+\pi^{0} \\
\pi^{+}+\pi^{+}+\pi^{-}
\end{array}\right.
\end{aligned}
$$

The mystery was that the two mesons were otherwise exactly the same as far we could tell (same mass, spins, decay rate, and so forth). Of course, at 
the time, it was assumed parity symmetry was always conserved, so the only acceptable solution must be that they are different particles. T.D. Lee and C.N. Yang realized, however, that no tests on weak-interaction parity symmetry had ever been carried out. In their groundbreaking paper [24], they proposed that the $\tau$ and $\theta$ mesons are actually the same thing and what was being observed was a possible parity violation. Lee and Yang suggested further experiments to confirm this.

Experimentalist C.S. Wu would set up the technically difficult task of confirming their hypothesis. In the experiment, $\mathrm{Wu}$ set up a film of radioactive cobalt-60 atoms that decay according to the following reaction:

$$
{ }^{60} \mathrm{Co} \rightarrow{ }^{60} \mathrm{Ni}+e+\overline{v_{e}},
$$

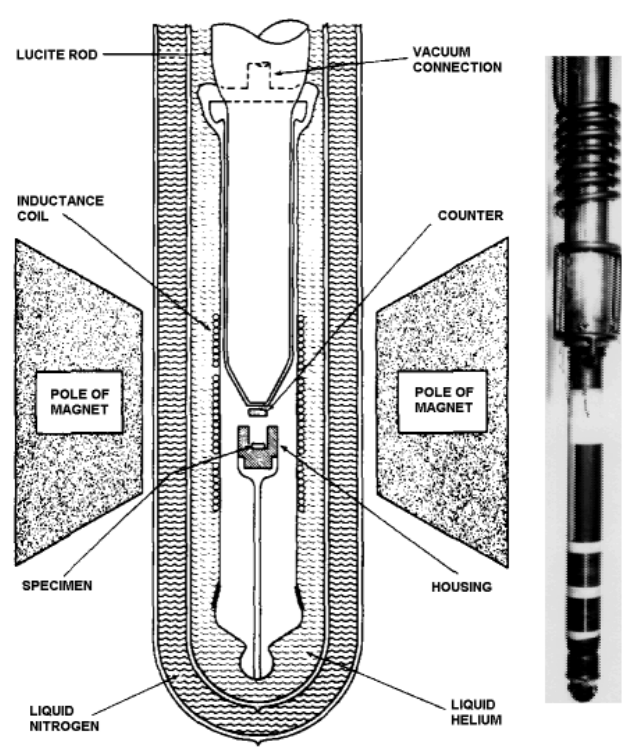

Figure 2.3: Schematic alongside actual photo of famous $\mathrm{Wu}$ experiment on cobalt-60 $\beta$ decay.

Cobalt-60 undergoes beta $(\beta)$ decay-emitting electrons and gamma $(\gamma)$ rays as it turns into nickel. Essentially, the idea was to observe how the atoms decayed: in what direction did the $\beta$ and $\gamma$ emit from the atoms and whether or not it favored a particular direction. Cobalt-60 was chosen for the experiment because its high spin makes it susceptible to magnetic fields and it is possible to line up the atoms and get a uniform reading. The sample was then cooled to approximately $1.2^{\circ} \mathrm{K}$ to reduce energetic 
perturbations amongst the atoms, further increasing the ability to gather accurate readings. If the decay process favored one side, it would be a clue to parity violation.
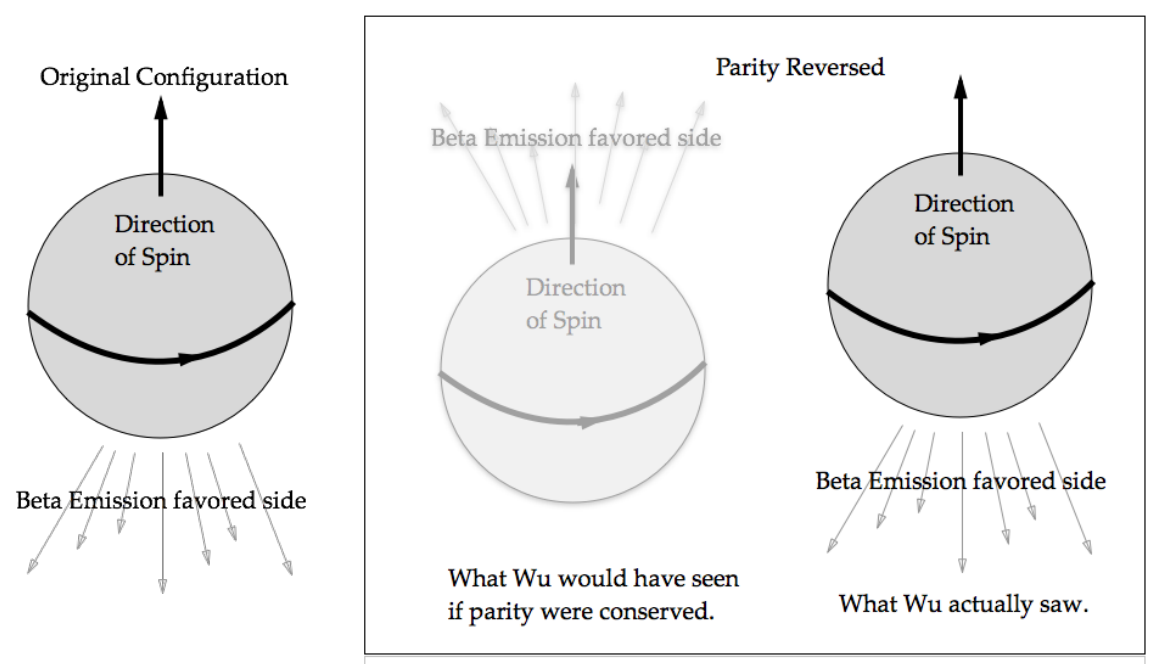

Figure 2.4: A simplified diagram describing possible results of Wu experiment. No matter what orientation of the experiment, $\beta$-decay always favored the side opposite to spin.

$\mathrm{Wu}$ found that the $\beta$-emissions favored the direction opposite of spin: $60 \%$ of the $\beta$-emissions occurred on that side while $40 \%$ were emitted at the other [32]. Moreover, she found that when the orientation of the experiment was reversed, $\beta$-emissions would still favor the side opposite to spin. Because $\beta$-emissions should be affected by magnetic fields (since they are electrons), this was was a clear indication of parity violation in weak interactions (Fig. 2.4).

It goes without saying that the physics community was shocked by the results. Ever since the Greeks had noticed and found pleasure in symmetries, it had been assumed this was a non-negotiable feature of Nature at all levels. In fact, prior to publication of the results, Pauli wrote, "I do not believe that the Lord is a weak left-hander, and I am ready to bet a very high sum that the experiments will give symmetric results [17]." If such a bet had been made, he would have lost as the experiment convincingly showed that Universe does have a bias and is very slightly left-handed. In later experiments, it would be found that parity is violated in all weak interactions. Not only was the "tau-theta puzzle" finally solved, but it 
appeared the mirror of symmetry was beginning to show cracks.

\subsection{The Fall of CP (Charge Conjugation/Parity) Sym- metry}

In an effort to salvage symmetry, it was suggested that while P-symmetry could be broken, CP (the combination of charge and parity) was still valid [30]. Charge conjugation involves multiplying the charge value of the particles in the interaction by -1 (e.g., electrons go from -1 to +1 charge). To wit, this means replace every particle with its antiparticle counterpart. To be applied to Wu's experiment, this would require the use of magnetic fields generated by positrons flowing in the opposite direction around anti-cobalt atoms. Unfortunately, for the foreseeable future, it is not technically feasible to create the antimatter version of such a massive atom. However, with the inclusion of antiparticles, theoretically at least, symmetry appeared to be on solid footing again.

The small victory would be short lived. Recall the "tau-theta puzzle" which originally led Lee and Yang to first question the validity of P-symmetry. Kaons would once again prove instrumental in providing evidence of symmetry breaking. In 1964, J.W. Cronin et. al. created an experiment to observe the decay rates of the neutral kaons:

$$
\begin{aligned}
& K_{S}^{0} \rightarrow \pi^{+}+\pi^{-} \\
& K_{L}^{0} \rightarrow \pi^{+}+\pi^{-}+\pi^{0}
\end{aligned}
$$

Where 'S' stands for short and ' $L$ ' stands for long-a reference to their decay rates with the ' $\mathrm{S}$ ' version having a rate three orders of magnitude shorter. In actuality, the neutral kaons can decay into various other forms, but what's important here, is that they decay into these two and three pion forms respectively. It turns out that a tiny fraction $\left(2.0 \pm 0.4 \times 10^{-3}\right)$ of the 'L' type neutral kaons actually decay into two pions as well. This result was a clear indication of CP symmetry [7]. Further observations of neutral kaon decay display clearer evidence of $\mathrm{CP}$ violations in that it has been found that decay rates emitting positrons occur slightly more preferentially than decay into electrons-indicating a $\mathrm{CP}$ violation between matter and antimatter (Eq. 2.4) [23]. 


$$
\frac{\operatorname{rate}\left(K_{L}^{0} \rightarrow e^{+}+\pi^{-}+v\right)}{\operatorname{rate}\left(K_{L}^{0} \rightarrow e^{-}+\pi^{+}+\bar{v}\right)} \approx 1.00648 \pm 3.5 \times 10^{-4}
$$

\subsection{Time and CPT (Charge Conjugation/Parity/Time) Symmetry}

Time symmetry is probably the most difficult to intuitively grasp since there is no real concept of it in the human experience. Essentially, it is idea that watching an event forwards or backwards in time should be indistinguishable. However, when physicists talk about time reversal, it is on a micro, not macro scale. Obviously, one could easily identify which was playing backwards with, say, a video of a glass of milk shattering on the ground. Probability makes it effectively impossible to produce an event that would look like shards of glass spontaneously gathering together with individual drops of milk coalescing back into the shape of a cup. Time reversal, in this sense, is meant to describe individual particle interactions: a pool ball hitting another on a table, for example. In this case, the event would appear to be perfectly acceptable physical events played in either direction.

After all this, what we are left with is statement of symmetry in the Universe in the form of CPT. The foundation of modern physics requires that CPT is conserved. But, if this is the case and we now know CP is not conserved, it turns out, $\mathrm{T}$ must also not be conserved. In the preceding kaon decay experiment conducted by Fitch, it was found that some CP obeying kaons decayed into an asymmetric state. If CPT symmetry must remain valid, the $\mathrm{CP}$ asymmetric kaons must regain symmetry under a $\mathrm{T}$ reflection. But in order to turn an asymmetric state into a symmetric one, $\mathrm{T}$ reflection itself must also be asymmetric [30].

Why does the Universe appear so nearly symmetric, but not quite? As stated earlier, in the vast majority of cases, symmetry is always conserved: it is only in the world of the exceedingly tiny weak interactions that we find violations. It is so unnoticeable, we only managed to find it in the past 50 years. Concerning this paucity of symmetry breaking phenomena in Nature, Feynman offered a rather poetic explanation:

There is a gate in Japan, a gate in Neiko, which is sometimes called by the Japanese the most beautiful gate in all Japan; it 
was built in a time when there was great influence from Chinese art. This gate is very elaborate, with lots of gables and beautiful carving and lots of columns and dragon heads and princes carved in the pillars, and so on. But when one looks closely he sees that in the elaborate and complex design along one of the pillars, one of the small design elements is carved upside down; otherwise the thing is completely symmetrical. If one asks why this is, the story is that it was carved upside down so that the gods will not be jealous of the perfection of man. So they purposefully put an error in there, so that the gods would not be jealous and get angry with human beings.

We might like to turn the idea around and think that the true explanation of the near symmetry of nature is this: that God made the laws only nearly symmetric so that we should not be jealous of His perfection! [15] 


\section{Chapter 3}

\section{Capturing Antimatter}

\subsection{Where is all the Antimatter?}

It seems then, there is a subtle asymmetry in Nature, and it is exceedingly small. CP asymmetry is so small in fact, it doesn't come close enough to explaining matter's utter dominance over antimatter we observe in the Universe today $[6,19,30]$. As discussed earlier, for all intents and purposes, antimatter is simply the mirror image of normal matter. If symmetries are the answer to this puzzle, it is as if we found a tiny imperfection in a cosmic quarter that should cause a nearly imperceptible favoring of heads over tails, only to find it almost always turns up heads.

Dirac's imagined antimatter stars and galaxies have never materialized. The search for antimatter in the Universe continues with several geosynchronous satellites including PAMELA and Alpha Magnetic Spectrometer02 (launched in 2006) currently scanning space for tell-tale signs of large amounts of antimatter such as emissions of $\gamma$-radiation pouring out at borders where matter annihilates with antimatter [21]. Although a few examples have been identified, such as the center of the Crab Nebula where a rapidly rotating neutron star is surrounded by a cloud of electrons and positrons (Fig. 3.1), or near the event horizons of black holes which act as antimatter factories where accretions of antimatter soup rotate at velocities nearing the speed of light [2], these are exceedingly rare astrophysical phenomena that make up only a tiny portion of the visible Universe.

Therefore, studying antimatter in Nature is in all practicality, impossible. It is simply out of the question to mine it from somewhere or observe it in any amount of detail from space. In fact, up until we created antihy- 


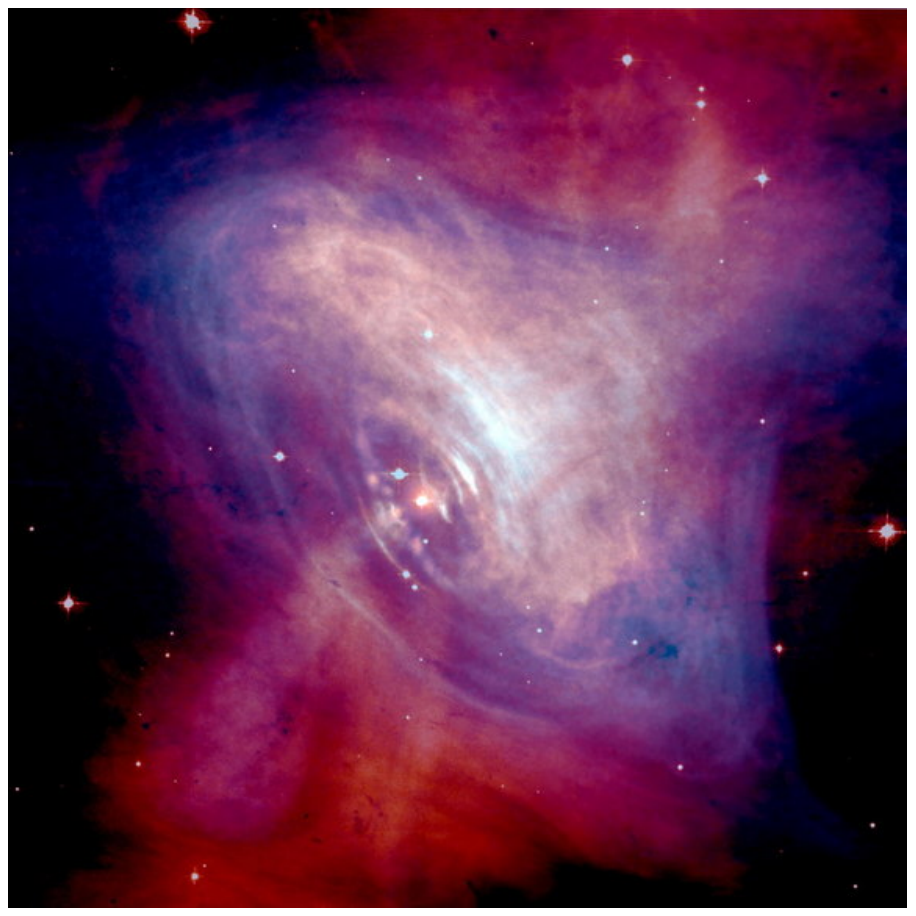

Figure 3.1: X-ray (blue) and optical (red) superimposed images of rapidly rotating pulsar in the heart of the Crab Nebula. The center ring is believed to be a mixture of matter and antimatter being propelled at near the speed of light. Image by NASA.

drogen $(\bar{H})$ in the past 20 or so years, it is possible that our corner of the Galaxy had never before seen anti-elements. Because of this, studying it has become a focal point of major experimental research where some of the world's grandest experiments are dedicated to artificially generating antiparticles for further study. Our current research is involved in such a endeavor.

\subsection{Technical Hurdles}

In order to answer questions about matter/antimatter asymmetry and determine whether or not CPT violation is a part of the explanation requires detailed study of antiparticles. Since Anderson's discovery of positrons in 1932, it has been possible to artificially generate small amounts of it. In fact, they are used extensively in medicine for both body scanning and disease treatment. For example, antiproton therapy to attack cancer cells 
is currently undergoing research [20], and positron emission tomography (PET) scans have become a commonplace tool used by medical professionals in the past decades. The speed of development in antiparticle research is directly related to the mass of the particle being studied. As positrons are about 2000 times lighter than antiprotons, creation of the latter requires much greater energies and hence, did not happen until powerful enough particle accelerators became technically feasible. Within the last 15 years, creation of antihydrogen has become a reality, albeit, still enormously complex and expensive to accomplish.

Of course, one of the problems in studying antimatter is that it has a tendency to annihilate upon interaction with matter. Thus, much of the technical challenge comes with trapping it in experiments that isolate it from everything else, including the very container it which it is being held. This requires the utilization of vacuums on the order of $10^{-11}-10^{-12}$ Torr [3] which is about as rare as the space surrounding the surface of the Moon. Magnetic and electric fields are utilized for containment preventing migration of the sample away from the central axes of the trap.

Finally, the collisions that produce antiprotons and positrons tend to generate particles with very high kinetic energies. The first experiments conducted in the 1990s produced antihydrogen at relativistic energies making it extremely difficult to study [6]. Because of this, novel methods have been devised to cryogenically cool the particles allowing for initial study. Needless to say, the combination of these issues pose some formidable technical challenges.

The Antiproton Decelerator (AD) at CERN (European Organization for Nuclear Research) has been conducting pioneering work on the formation of antiparticles and antihydrogen. Recent projects there in generating and capturing antihydrogen has revolved around containment devices such as Penning-Malmberg traps (Fig. 3.2). Essentially, cryogenic techniques are used to cool antiprotons and positrons which are then injected into either ends of the trap. From there, powerful superconducting magnets and electric fields provide containment for the newly formed antihydrogen atoms. Recent experiments including ATHENA, ALPHA (Antihydrogen Laser PHysics Apparatus) and ATRAP (Antihydrogen Trap) collaborations, have managed to capture antihydrogen for several minutes at temperatures on the order of $0.5^{\circ} \mathrm{K}$ allowing for initial study into the structure of the atom and the beginnings of gravitational experiments. However, samples with even lower kinetic energy are desired for even greater detailed study. 


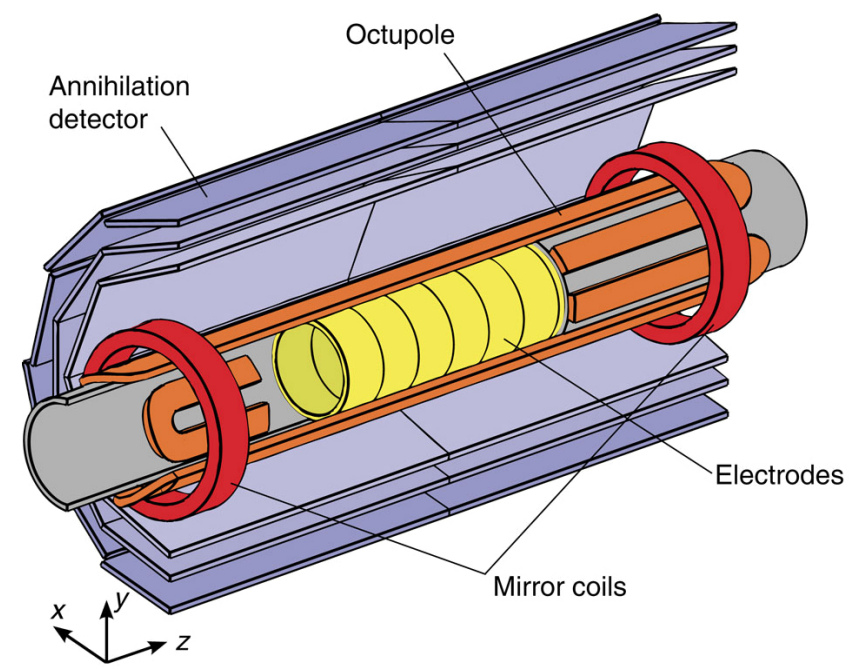

Figure 3.2: Diagram of trapping region of ALPHA experiment that synthesizes antihydrogen atoms. Penning trap is in central portion in yellow. Antiprotons and positrons are injected from either ends of the trap to allow mixing and formation of antihydrogen. The Penning trap has a diameter of about $44.5 \mathrm{~mm}$ (drawing is not to scale).

\subsection{Current Work}

To meet these goals, new collaborations including ALPHA2 and GBAR have been assembled with the goal of producing $\bar{H}$ in the $\mathrm{mK}$ range. This requires innovative approaches as we near the limits of current methods. Thus, present work has been focused on the production of antihydrogenions $\left(\bar{H}^{+}\right)$to be used as an intermediary for sympathetic cooling methods. The idea is to use laser-cooled, charged particles (such as $\mathrm{Be}^{+}$) to bleed off kinetic energy from the $\bar{H}^{+}$sample. Since $\bar{H}^{+}$carries an unscreened charge, they are susceptible sympathetic cooling methods unlike the electrically neutral $\bar{H}$. The GBAR collaboration envisioned producing small quantities of $\bar{H}^{+}$, but their proposed method would have yielded less than one part per 60 million reactions. This low yield motivated the search for a more efficient method in our current study.

As $\bar{H}$ can be trapped for fairly extended periods of time, it may be possible to utilize positron-antihydrogen $\left(e^{+}+\bar{H}\right)$ reactions to produce the ion $\bar{H}^{+}$. The inverse of this reaction, the photodetachment of an electron $\left(1 s^{21} S^{e}\right)$ from a hydrogen ion, 


$$
H^{-}+h v \rightarrow H+e^{-},
$$

has been of interest to astrophysicists for decades as it is known to cause the opacity of stellar bodies including the Sun. This process is very well understood and has been studied extensively both through theory and observation [28,31]. Hence, it is possible to create an accurate theoretical model. Exploiting charge conjugation and time-reversal symmetry, it is possible to generate a theoretical model of the inverse process that would create $\bar{H}^{+}$by the following reaction:

$$
\bar{H}(1 s)+e^{+} \rightarrow \bar{H}^{+}\left(1 s^{21} S^{e}\right)+h v .
$$

Exploiting Ohmura and Ohmura's effective range theory including earlier work from Bethe and Longmire, a model for the cross section of the photodetachment process has been generated that shows excellent agreement with available observational and experimental data (Fig. 3.3).

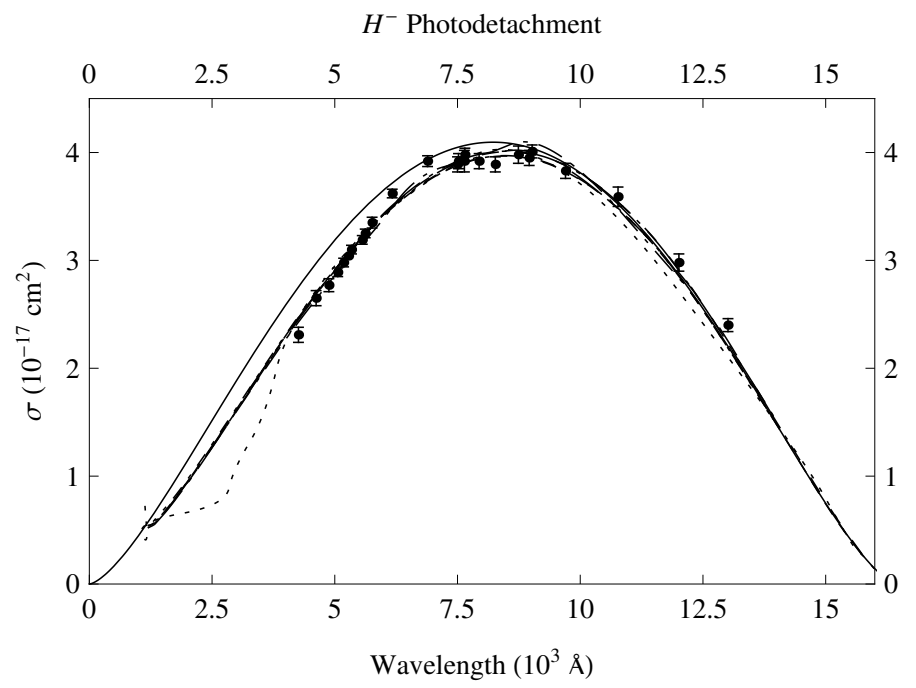

Figure 3.3: Dots are experimental values from Smith and Burch, while the solid line gives theoretical results derived from Ohmura and Ohmura's effective range theory

Calculations for the inverse reaction, radiative attachment of a positron to $\bar{H}$ (Eq. 3.2), have been generated along with a temperature dependent cross section for the generation of $\bar{H}^{+}$. Positron temperatures used to form antihydrogen are in the range of $10 \mathrm{~K}$ or lower where the cross section varies with $\sqrt{T_{e}}$ (Fig 3.4). In the temperature range of interest (sub 
$1 \mathrm{~K}$ range), the cross section is on the order of $10^{-23} \mathrm{~cm}^{2}$ indicating low yields and hinting at a high level of difficulty in setting up the experiment. Appendix $C$ provides more detailed information on the derivation of the cross products of the photodetachment and radiative attachment processes. Further work is currently underway to possibly improve yields.

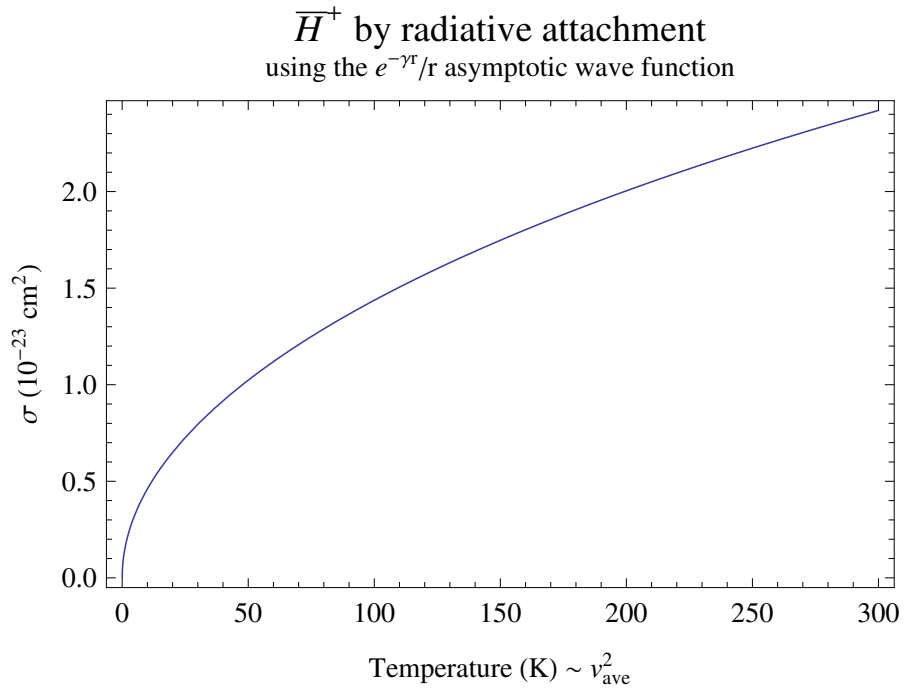

Figure 3.4: Cross section for radiative attachment of second positron forming $\bar{H}^{+}$ 


\section{Chapter 4}

\section{Continuing Search for CPT Asymmetry}

\subsection{CPT Tests and the Consensus Thus Far}

The ability to artificially generate small quantities of anti-atoms at cryogenic energies has finally provided an opportunity to experimentally test and verify CPT symmetry. Most recent experiments have dealt with trying to find characteristic differences between particles and antiparticles including differences in mass, spin, magnetic moments and so forth. A recent experiment with the ATRAP group at CERN utilized double Penning traps to compare magnetic moments between protons and antiprotons and found results consistent with the Standard Model and CPT accurate to 5 parts per million $\left(5 \times 10^{-6}\right)$ [11]. Also, work in 2007 with the ASACUSA collaboration pushed differences between proton and antiproton charge and mass to 2 parts per billion $\left(2 \times 10^{-9}\right)$ [21]. In short, at the precision available today, no difference has been found between particles and antiparticles-as far as we can tell, they are mirror images of each other and CPT holds.

It seems, therefore, that given current limitations, CPT is on solid theoretical and experimental footing. If CPT violations are found, "all hell breaks loose," Griffiths has quoted an unnamed theorist as saying [19]. Indeed, CPT symmetry is so fundamental to our understanding of the Universe and the framework of modern physics, that its violation would require radical rethinking and could be an upheaval more significant than the discovery of parity violation in weak interactions by Lee, Yang, and 
$\mathrm{Wu}$. To put it another way, it has been stated that CPT symmetry is guaranteed under current quantum field theories (QFTs) and that their violation would force a fundamental change in our understanding of Nature [16].

\subsection{Future Studies}

The discussion, however, is far from over. Tantalizing theoretical work by Bluhm, Fujiwara, and others have pointed to possible future experimental tests that could uncover CPT violations $[4,5,16]$. Their work has focused on energies relative to the Planck scale and described by the standard-model extension (SME) which in general, is beyond the sensitivity of current experiments. In particular, interest has been focused on spectroscopic comparison between hydrogen and antihydrogen in the $1 S-2 S$ and hyperfine transitions from ground state.

Bluhm, in particular, has proposed theories that involve CPT breaking terms in hyperfine transitions but would require experiments that are approximately three orders of magnitude more accurate than those run to date. Cooling in the $\mathrm{mK}$ range, as described in this paper, may aid in uncovering these violations. Other possibilities he has suggested, however, would require ultra cooling on the order of $100 \mu \mathrm{K}$ which is about 3 orders of magnitude colder than the experiments proposed utilizing antihydrogen ions and sympathetic cooling. More recent work by Bluhm points to CPT breaking observations that may occur when accounting for sidereal time variations that would require exceedingly precise and complicated experiments that would have to compensate for the spin of the Earth and its motion through space [4]. Although difficult, it is by no means beyond present capabilities.

\subsection{Conclusion}

In under a century, we've gone from discovering antimatter with the brilliant theoretical work of Dirac, to experimentally probing the deepest unanswered questions of the Universe with regards to its symmetry. This has also answered (and raised) questions regarding baryogenesis and what we see today with a Universe dominated by ordinary matter. For most of human history, symmetry was accepted as a non-negotiable law and a statement of the way the Universe works according to our best under- 
standing. The fact that this accepted law was abruptly overturned a mere 30 years following Dirac's momentous work should bring some level of solace to physicists today continuing to ask these questions. Despite current consensus in CPT infallibility, hell has broken loose before, following reformulation of theories, and further questions down the road. 


\section{Appendix A}

\section{The Dirac Equation: How Matrices Save the Day}

To simplify things, we will derive the one-dimensional case. The threedimensional version is basically the same but leads to more complex $4 \times 4$ matrices. To begin, we'll recapitulate the relativistic energy equation:

$$
E^{2}=p^{2} c^{2}+m_{0}^{2} c^{4}
$$

Essentially, Dirac wanted a first order differential equation, but to do this, he had to get rid of the squared energy. Dirac accomplished this by introducing additional variables:

$$
E^{2}=\left(\alpha p c+\beta m_{0} c^{2}\right)^{2}
$$

Which expands out to,

$$
E^{2}=\alpha^{2} p^{2} c^{2}+\alpha \beta p m_{0} c^{3}+\beta \alpha p m_{0} c^{3}+\beta^{2} m_{0}^{2} c^{4} .
$$

Now, what we want here is for Eq. A.3 to look like Eq. A.1. In order for that to happen, the following has to be true:

$$
\begin{aligned}
& \alpha^{2}=\beta^{2}=1 \\
& \alpha \beta+\beta \alpha=0
\end{aligned}
$$

No need to struggle with a solution here: no real numbers will simultaneously satisfy the above conditions. Dirac realized the only way through 
the impasse was to utilize matrices. Thus, he set $\alpha$ and $\beta$ equal to the following $2 \times 2$ matrices:

$$
\alpha=\left(\begin{array}{ll}
0 & 1 \\
1 & 0
\end{array}\right), \beta=\left(\begin{array}{cc}
1 & 0 \\
0 & -1
\end{array}\right) .
$$

These matrices have the properties we are looking for:

$$
\alpha^{2}=\left(\begin{array}{ll}
0 & 1 \\
1 & 0
\end{array}\right)^{2}=\left(\begin{array}{ll}
1 & 0 \\
0 & 1
\end{array}\right), \beta^{2}=\left(\begin{array}{cc}
1 & 0 \\
0 & -1
\end{array}\right)^{2}=\left(\begin{array}{ll}
1 & 0 \\
0 & 1
\end{array}\right)
$$

with,

$$
\alpha \beta=\left(\begin{array}{cc}
0 & -1 \\
1 & 0
\end{array}\right), \beta \alpha=\left(\begin{array}{cc}
0 & 1 \\
-1 & 0
\end{array}\right)
$$

thus,

$$
\alpha \beta+\beta \alpha=\left(\begin{array}{ll}
0 & 0 \\
0 & 0
\end{array}\right) .
$$

Hence, Dirac had figured out a way to make his equation first order in time. 


\section{Appendix B}

\section{Noether Theorem: Linear One-Dimensional Case}

Here, we will use the case of symmetry in translation of one-dimensional motion and show how it implies conservation of linear momentum. In essence, this provides a mathematical justification for the physical observation that moving a particle from one position to another does not alter its momentum. This is known as symmetry of translation. Although these generally only apply to Lagrangian and Hamiltonian equations, they are extremely common in physics thus its usefulness is pervasive.

Consider a particle moving along a one-dimensional line, with the Lagrangian, $\mathscr{L}(x, \dot{x})=K-V$. Taking the partial derivatives with respect to $x$ and $\dot{x}$, we get equations for force and momentum respectively,

$$
\frac{\partial \mathscr{L}}{\partial x}=-\frac{\partial U}{\partial \dot{x}}=F, \quad \frac{\partial \mathscr{L}}{\partial \dot{x}}=m \dot{x}=p
$$

Now, taking the time derivative of the momentum gives us,

$$
\frac{d}{d t} \frac{\partial \mathscr{L}}{\partial \dot{x}}=\dot{p}=m a=F
$$

Combining B.1 and B.2 quickly leads to the Euler-Lagrange equation:

$$
\frac{\partial \mathscr{L}}{\partial x}=\frac{d}{d t} \frac{\partial \mathscr{L}}{\partial \dot{x}}
$$

Which is just a recapitulation of Newton's second law: $F=m a$. 
Now, suppose I have some transformation, $x(s)$, that moves the particle from one position to another. Then, we make the claim that such a translation makes no change in the Lagrangian. Or more compactly,

$$
\frac{d}{d s} \mathscr{L}(x(s), \dot{x}(s))=0
$$

Let us assume $C=p * d x(s) / d s$ is a conserved quantity. Which, with the product rule, we mean to say,

$$
\dot{C}=\dot{p} \frac{d x(s)}{d s}+p \frac{d \dot{x}(s)}{d s}=0
$$

Replace the $\dot{p}$ and $p$ with our earlier Lagrangian derivations:

$$
\dot{C}=\frac{\partial \mathscr{L}}{\partial x} \frac{d x(s)}{d s}+\frac{\partial \mathscr{L}}{\partial \dot{x}} \frac{d \dot{x}(s)}{d s}
$$

Using the chain rule, we can recognize that the right side of equation B.6 equals the left side of B.4,

$$
\frac{\partial \mathscr{L}}{\partial x} \frac{d x(s)}{d s}+\frac{\partial \mathscr{L}}{\partial \dot{x}} \frac{d \dot{x}(s)}{d s}=\frac{d}{d s} \mathscr{L}(x(s), \dot{x}(s))=0
$$

Thus proving the original claim that linear momentum is conserved (invariant) under symmetry of translation. The three-dimensional derivation is similar if somewhat more complicated. 


\section{Appendix C}

\section{Photodetachment and Radiative Attachment}

\section{C.1 Photodetachment}

The cross section for the photodetachment of one electron from the $1 s^{2}{ }^{1} S^{e}$ state of $\mathrm{H}^{-}$involves the corresponding (velocity gauge) dipole transition operator (in atomic units $\hbar=m_{e}=e=1$ ).

$$
\sigma_{V}=\frac{4 \alpha a_{0}^{2}}{3} \frac{p}{2 \omega}\left(\int \Psi^{*}\left(\overrightarrow{r_{1}}, \overrightarrow{r_{2}}\right)\left(\frac{\partial}{\partial z_{1}}+\frac{\partial}{\partial z_{2}}\right) \Psi_{i}\left(\overrightarrow{r_{1}}, \overrightarrow{r_{2}}\right) d^{3} r_{1} d^{3} r_{2}\right)^{2}
$$

Where $p$ is the momentum of the detached electron and $\omega$ is the angular frequency of the incoming photon. For the final state, a plane wave is utilized:

$$
\psi_{f}^{*}(\vec{r})=e^{-i \vec{k} \cdot \vec{r}} .
$$

Note that only the p-state portion of the outgoing electron plane wave will contribute. In this approximation, the cross section becomes,

$$
\sigma_{V}=\frac{4 \alpha a_{0}^{2}}{3} \frac{k}{k^{2}+\gamma^{2}}\left(\int e^{-i \vec{k} \cdot \vec{r}}\left(-i \frac{1}{k} \vec{k} \cdot \nabla\right) \psi_{i}(r) d^{3} r\right)^{2} .
$$

Finally, using current values of the Bohr radius $a_{0}$ and fine structure constant $\alpha=1 / c$, we obtain, 


$$
\sigma_{P H}=\frac{0.68983 \times 10^{-17} \mathrm{~cm}^{2}}{1-\rho \gamma} \frac{1}{\gamma^{2}}\left(\frac{\lambda}{\lambda_{0}}\right)^{3 / 2}\left(1-\frac{\lambda}{\lambda_{0}}\right)^{3 / 2}
$$

\section{C.2 Radiative Attachment}

The radiative attachment of an positron to $\bar{H}$ forming $\bar{H}^{+}$is the inverse process of the photodetachment process described above. Utilizing the principle of detailed balance, we arrive at,

$$
\sigma_{R A}=\frac{g_{1} p_{1}^{2}}{g_{2} p_{e}^{2}} \sigma_{P H}
$$

where $g_{j}$ is the statistical weight. The final state has,

$$
g_{1}=\text { Polarizations } \times\left(2 S_{\text {electron }}+1\right)(2 L+1)=6,
$$

and $p_{1}$ is the photon momentum relative to the ion:

$$
p_{1}=p \omega=\frac{E_{\omega}}{c}=\frac{\hbar \omega}{c}=\frac{k^{2}+\gamma^{2}}{2 c},
$$

where the final step utilizes energy conservation. The initial state has,

$$
g_{2}=\left(2 S_{\text {electron }}+1\right)\left(2 S_{\text {target } 2}+1\right)(2 L+1)=12,
$$

and $p_{2}$ is the positron momentum $k$. In atomic units, $c$ is the inverse of the fine structure constant $\alpha$, so in terms of incoming positron energy $E_{e}=1 / 2 k^{2}$,

$$
\sigma_{R A}=\frac{.429304 \times 10^{-17} \mathrm{~cm}^{2}}{2^{2} \times 137.036^{2}} \frac{\sqrt{2 E_{e}}}{2 E_{e}+\gamma^{2}} .
$$




\section{Bibliography}

[1] G.B. Andresen, M.D. Ashkezari, and M Baquero-Ruiz. Trapped antihydrogen. Nature, 468(7324):673-6, December 2010.

[2] Cosimo Bambi, Alexander D Dolgov, and Alexey A Petrov. Black holes as antimatter factories. arXiv, (1):1-5, 2013.

[3] P Belochitskii. STATUS OF THE ANTIPROTON DECELERATOR AND OF THE ELENA PROJECT AT CERN. In COOL 2007, pages 6-10, 2007.

[4] Robert Bluhm. Lorentz and CPT tests in matter and antimatter. $\mathrm{Nu}$ clear Instruments and Methods in Physics Research, Section B: Beam Interactions with Materials and Atoms, 221(1-4):6-11, July 2004.

[5] Robert Bluhm, V. Kostelecký, and Neil Russell. CPT and Lorentz Tests in Hydrogen and Antihydrogen. Physical Review Letters, 82(11):22542257, March 1999.

[6] M. Charlton, S. Jonsell, L. V. Jø rgensen, N. Madsen, and D. P. van der Werf. Antihydrogen for precision tests in physics. Contemporary Physics, 49(1):29-41, January 2008.

[7] J.H. Christiansen, J.W. Cronin, V.L. Fitch, and R Turlay. Evidence for the $2 \pi$ Decay of the K Meson. Physical Review Letters, 1(4):138-140, 1964.

[8] Frank Close. Antimatter. Oxford University Press, New York, 2009.

[9] P.A.M. Dirac. A Theory of Electrons and Protons. Proceedings of the Royal Society A: Mathematical, Physical and Engineering Sciences, 126(801):360-365, January 1930. 
[10] P.A.M. Dirac. Theory of electrons and positrons, 1933.

[11] J. DiSciacca, M. Marshall, K. Marable, G. Gabrielse, S. Ettenauer, E. Tardiff, R. Kalra, D. Fitzakerley, M. George, E. Hessels, C. Storry, M. Weel, D. Grzonka, W. Oelert, and T. Sefzick. One-Particle Measurement of the Antiproton Magnetic Moment. Physical Review Letters, 110(13):130801, March 2013.

[12] Albane Douillet, Paul Indelicato, Sebastian Wolf, and Ferdinand Schmidt Kaler. Preparing single ultra-cold antihydrogen atoms for the free-fall in GBAR. arXiv.org, 2014.

[13] Albert Einstein. Obituary: Emmy Noether. New York Times, May 1935.

[14] Graham Farmelo. The Strangest Man: The Hidden Life of Paul Dirac, Mystic of the Atom. Basic Books, New York, NY, 2009.

[15] Richard P. Feynman. Six Not-So-Easy Pieces. Addison-Wesley Publishing Company, Inc., Pasadena, California, 1997.

[16] Makoto C Fujiwara. Antihydrogen, cpt, and naturalness. arXiv, (1):1$5,2013$.

[17] Martin Gardner. The New Ambidextrous Universe. W.H. Freeman and Company, New York, NY, 3rd edition, 1990.

[18] W.B. Gibson and B.R. Pollard. Symmetry Principles in Elementary Particle Physics. Cambridge University Press, London, England, 1976.

[19] David Griffiths. Introduction to Elementary Particles. WILEY-VCH Verlag GmbH \& Co., Weinheim, 1st edition, 2008.

[20] M Hori, J Walz, and Johannes Gutenberg-universit. Physics at CERN's Antiproton Decelerator. arXiv, 2013.

[21] Dezso Horváth. Testing CPT invariance with antiprotonic atoms. Radiation Physics and Chemistry, 76(3):397-403, 2007.

[22] T.D. Lee. Elementary Particles. Physics Today, 13(10):30, 1960.

[23] T.D. Lee. Symmetries, Assymetries, and the World of Particles. University of Washington Press, Seattle and London, 1988. 
[24] T.D. Lee and C.N. Yang. Question of Parity Conservation in Weak Interactions. Physical Review, 104(1):254-258, 1956.

[25] T.D. Lee and C.N. Yang. Parity Nonconservation and a TwoComponent Theory of the Neutrino. Physical Review, 105(5):226-227, 1957.

[26] David Oliver. The Shaggy Steed of Physics. Springer-Verlag, New York, 1994.

[27] J. Robert Oppenheimer. On the Theory of Electrons and Protons. Physical Review Letters, 35:562-563, 1930.

[28] Stephen J. Smith and David S. Burch. Relative Measurement of the Photodetachment Cross Section for H-. Physical Review, 116(5):1125-1131, 1959.

[29] John R. Taylor. Classical Mechanics. University Science Books, Bolder, Colorado, 2005.

[30] Eugene P. Wigner. Violations of Symmetry in Physics. Scientific American, 213(6):28-36, December 1965.

[31] A.W. Wishart. The bound-free photodetachment cross section of H-. Journal of Physics B: Atomic, Molecular and Optical Physics, 3511, 1979.

[32] C.S. Wu, F. Ambler, Hayward W., D.D. Hoppes, and R.P. Hudson. Experimental Test of Parity Conservation in Beta Decay. Physical Review, 105(1413):1-3, 1957.

[33] C.N. Yang. Present Knowledge About the New Particles. Reviews of Modern Physics, 29(1):231-235, 1957. 